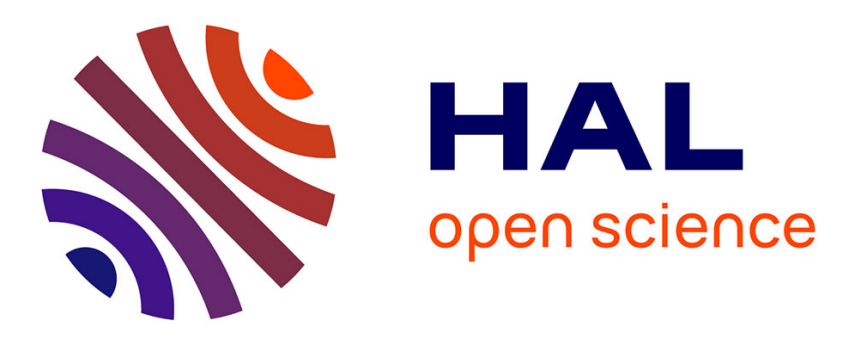

\title{
Fast monostatic scattering computation based on Gaussian beam shooting and frame decomposition
}

Christine Letrou, Mossaab Hariz, Barak Galanti, Amir Boag

\section{To cite this version:}

Christine Letrou, Mossaab Hariz, Barak Galanti, Amir Boag. Fast monostatic scattering computation based on Gaussian beam shooting and frame decomposition. COMCAS 2021: IEEE International Conference on Microwaves, Antennas, Communications and Electronic Systems, IEEE, Nov 2021, Tel-Aviv, Israel. pp.51-54, 10.1109/COMCAS52219.2021.9629065 . hal-03456099

\section{HAL Id: hal-03456099 https://hal.science/hal-03456099}

Submitted on 29 Nov 2021

HAL is a multi-disciplinary open access archive for the deposit and dissemination of scientific research documents, whether they are published or not. The documents may come from teaching and research institutions in France or abroad, or from public or private research centers.
L'archive ouverte pluridisciplinaire HAL, est destinée au dépôt et à la diffusion de documents scientifiques de niveau recherche, publiés ou non, émanant des établissements d'enseignement et de recherche français ou étrangers, des laboratoires publics ou privés. 


\section{Fast Monostatic Scattering Computation Based on Gaussian Beam Shooting and Frame Decomposition}

\author{
Christine Letrou \\ SAMOVAR Lab. \\ Télécom SudParis, IP Paris \\ Evry, France \\ Mossaab Hariz \\ Télécom SudParis, IP Paris \\ Evry, France \\ mossaab.hariz@telecom-sudparis.eu
}

christine.letrou@telecom-sudparis.eu

Amir Boag

School of Electrical Engineering

Tel Aviv University

Tel Aviv, Israel

boag@tauex.tau.ac.il

\author{
Barak Galanti \\ School of Electrical Engineering \\ Tel Aviv University \\ Tel Aviv, Israel \\ barakg@tauex.tau.ac.il
}

\begin{abstract}
Frame theory applied to source field decomposition facilitates the decomposition of an incident plane wave into a set of paraxial Gaussian beams. Spatial and spectral localization properties of such beams allow to bounce them through multiple reflections when reflecting surfaces are smooth and large enough as compared to wavelength. Gaussian beam spectral localization also yields dramatic reduction of the number of beams contributing to monostatic scattering cross section. An algorithm taking advantage of those properties is presented and applied to scattering by a set of blocks simulating an urban-like environment, with large dimensions as compared to wavelength. Numerical results will illustrate the method efficiency and will be compared to Fast Iterative Physical Optics results.

Index Terms-Gaussian beam, Gabor frame, monostatic scattering, Physical Optics
\end{abstract}

\section{INTRODUCTION}

The Monostatic Scattering Cross Section (SCS) of a given object or environment is defined on a grid of directions $\gamma$ as :

$$
S C S(\gamma)=20 \log _{10}\left(r_{o b s}\left|\varphi_{s}(\gamma) / \varphi_{i}(-\gamma)\right|\right)
$$

where $\varphi_{s}(\gamma)$ is the scattered far field propagating in the direction $\gamma$ at distance $r_{o b s}$ when a PW propagating in the opposite direction, with its field denoted $\varphi_{i}(-\gamma)$, is incident on the scattering object or environment. Calculating wave interactions for all directions of incidence is computationally intensive, especially when the problem is of large electrical size, defined by the large parameter $N=(k R)^{2}$ with $k$ being the wavenumber and $R$ the radius of the smallest circumscribing sphere. The number of scattering directions, in order to ensure sufficient sampling varies as $O(N)$ for scattering from/into all aspect directions (2D grid), and as $O\left(N^{1 / 2}\right)$ for scattering from/into azimuth directions only (1D grid).

Gaussian beam shooting (GBS) is well suited for problems involving multiple reflections, due to the spatial and spectral limited extents of paraxial Gaussian beams (GB). This method is thus a good candidate for calculations of SCS by large environments involving internal reflections. Frame based

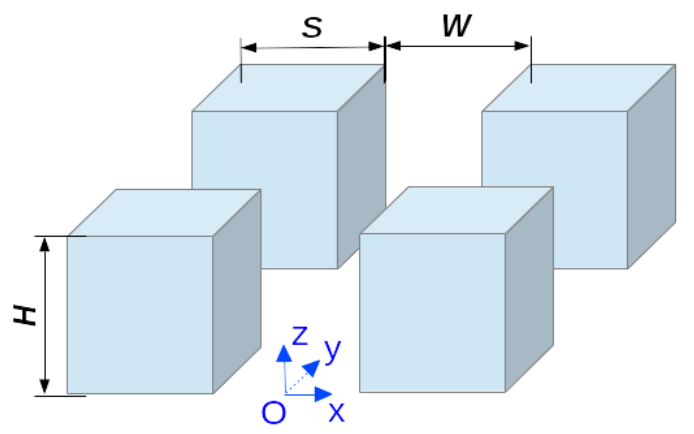

Fig. 1. Urban-like scattering environment.

decomposition provides full representation of incident fields in the form of Gaussian beam summations [1]. In this work, the simplest possible algorithm is proposed and tested, relying on paraxial GB properties.

The second section presents the basics of the frame based GBS method. The third one presents the application of this method to monostatic SCS computations in urban-like scenarios as presented in Fig. 1, and specific features of the proposed algorithm. Numerical results are presented in Section IV to illustrate the performance of the algorithm, and obtained results are compared to Fast Iterative Physical Optics (FIPO) [2] ones in order to determine the conditions of validity of the proposed algorithm.

\section{FRAME BASED GAUSSIAN BEAM SHOOTING}

\section{A. Gabor frames and frame decomposition}

A Gabor frame in $L^{2}(\mathbb{R})$ is a set of Gaussian functions obtained by translations of a "mother" Gaussian window in spatial and spectral domains, and satisfying the following oversampling property: "The product of translation steps in spatial and spectral domain is strictly less than $2 \pi$ ". This oversampling condition is necessary and sufficient for such 
a set to be complete. The mother window is given by: $w(x)=\sqrt{\sqrt{2} / L} e^{-\pi \frac{x^{2}}{L^{2}}}$, with $L$ being the "width parameter". Planar source field distributions can be decomposed into a two-variable Gabor frame, obtained as the product of two Gabor frames in $L^{2}(\mathbb{R})$. Each of the frame windows of such a frame will be denoted $w_{\mu}(x, y)$ with $\boldsymbol{\mu}=(m, n, p, q) \in \mathbb{Z}^{4}$, $(m, p)$ the translation indices in spatial domain along the $x$ and $y$ variables, $(n, q)$ the corresponding translation indices in spectral domain.

For a given planar source distribution, the set of decomposition coefficients on a given Gabor frame is not unique. The "minimum energy" set of coefficients is obtained by projection of the source field on a "dual frame". The dual frame windows are proportional to the initial frame windows for high enough oversampling, which will be the case in this work.

\section{B. Gaussian beam shooting and bouncing}

Gabor frame windows radiate in the form of Gaussian beams. The beam axes originate at the centers of the frame windows, defined by the $(m, p)$ indices, and their axis directions are defined by the window spectral translations, hence by the $(n, q)$ indices. If the width parameter $L$ is large enough as compared to the wavelength, the beam is "paraxial", which means that its spectrum is concentrated enough around its axis direction in order to limit divergence, at least up to a propagation distance equal to a few times the collimation distance $b=L^{2} / \lambda$.

If a Gaussian beam is incident on a planar surface, and if its footprint down to an arbitrarily small threshold level, is not intersecting the surface edges, it is transformed as if incident on an infinite planar surface. Its reflection is then easily represented via its plane wave spectrum transformation. If the beam is paraxial, the reflection operator is considered as well approximated by its value along the incident beam axis direction. Thanks to these properties, each beam radiated by a frame window is reflected in the form of a beam radiated by an "image frame" window defined in an "image plane". Bouncing beams consists of determining image frames corresponding to successive reflections, and which source windows are reflected along each succession of reflecting surfaces or "path".

\section{ApPliCATION TO MONOSTATIC SCATTERING}

\section{A. GBS initialization and approximations}

Decomposition coefficients of a plane wave on a Gabor frame in a given source plane can be derived in analytical form, by "projection" on frame windows, in the spectral domain, of the translated delta distribution corresponding to the plane wave direction of propagation. In order to test the validity of the simplest possible GBS algorithm, we shall use these coefficients to initialize the GBS algorithm from source frames defined on the sides of the smallest parallelepipedic box containing the scattering environment.

Beams are issued from frames defined in planes containing a box side. If beam origins are on a wall, beams are considered as reflected by the wall. If their origin is between two buildings, beams are launched into the environment, and bounced on internal walls. The same approximation is used inside the environment, considering that beams with their axes impinging on a given wall are reflected by this wall, even if their field along the edge is not negligible. If the beam axes do not impinge on a wall, the beams are considered as propagating in free space.

These approximations greatly simplify the algorithm, and contribute to minimize the computation time. Diffraction effects caused by environment discontinuities along building corners are however not accurately accounted for. Source fields on walls or between buildings are not correctly represented. The smaller the window width parameter $L$, the less inaccurate the initial field representation, yet the smaller the beam collimation distance, and hence the more diverging the beam, and the more inaccurate the approximation for beam propagation and bouncing inside the environment. Numerical tests are thus necessary to define the range of validity of this fast and simple algorithm.

\section{B. Algorithm and beam elimination}

The algorithm is divided into two parts. The first one leads to a "beam transfer matrix": each source beam contributing to monostatic scattering is transformed into an image beam multiplied by a product of paraxial reflection operators. The final result is thus given, for each exterior box side, by a list of $\boldsymbol{\mu}$ indices of source beams, associated with the information about the "path" followed by the beam (number of reflections or final reflection coefficient, final image plane).

Not all source beams contribute to monostatic scattering. For a beam to contribute, the opposite of at least one of the directions included in the source beam spectrum must belong to the spectrum of the final beam emerging from the environment. This condition leads to elimination of most of the beams.

The second part of the algorithm performs field calculation for all directions in a grid of scattering directions. To this end, for each of these directions the algorithm goes through a loop on all possibly contributing source windows, i.e. with their spectrum containing the opposite of this direction. Each of these beams is transformed into a scattered beam, and it will contribute to monostatic scattering only if the considered scattering direction is included in the spectrum of this scattered beam. This new condition, again related to the spectral localization of Gaussian beams, leads to a reduction of the number of beam field calculations.

\section{NUMERICAL RESULTS}

\section{A. GBS computations}

Test scenarios will involve different numbers of blocks, with hard boundary surfaces. Incident waves will be considered as scalar, which simplifies the algorithm without consequence on the conclusions which can be drawn from the test computations. We present here results for the $2 \times 2$ "buildings" scenario of Fig. 1. "Building" height and sides are respectively of length $H=24 \mathrm{~m}$ and $S=25 \mathrm{~m}$. The "street" width is $W=20 \mathrm{~m}$. Frequencies of interest should be high enough to make the 


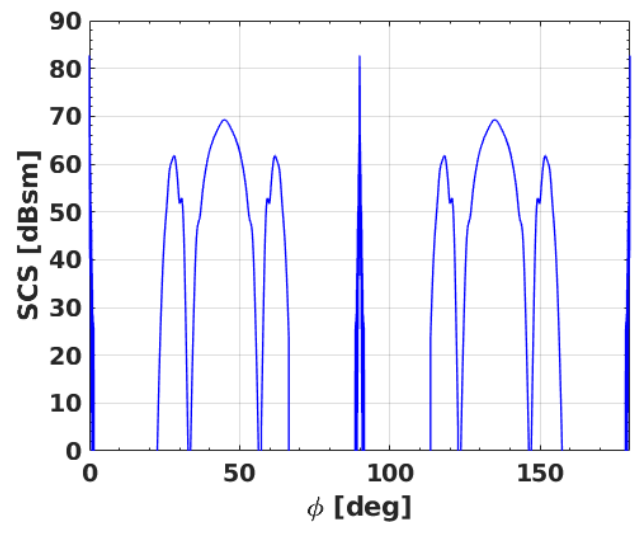

Fig. 2. Monostatic SCS for $\lambda=0.1 \mathrm{~m}$.

problem computationally intensive, and difficult to address by Physical Optics. SCS is computed for a grid of azimuthal directions (half-circle).

Fig. 2 presents the monostatic SCS computed at the $0.1 \mathrm{~m}$ wavelength with the simple GBS algorithm presented in the previous sections. The frame window width parameter $L$ was taken equal to $40 \lambda=4 \mathrm{~m}$, and the frame oversampling was of 2 along each Cartesian variable in a given plane. With these parameters, the initial number of frame windows, before beam elimination, is of the order of $10^{7}$. Beam elimination in the first part of the algorithm ("beam transfer matrix" computation) reduces the number of contributing source frame windows to about $10^{4}$. Finally, $10^{3}$ beams or less are contributing to scattered field into a given direction.

Results obtained for direct reflection by one of the exterior "walls" can be compared to an analytical solution. Fig. 3 presents such a comparison at the $0.01 \mathrm{~m}$ wavelength. Reflection occurs on the wall surface where the incident field is decomposed into frame windows, hence incident and reflected fields, for each initial frame window, are in the same plane, and there is no beam propagation. Thus, the smaller the frame window width parameter $L$, the more accurate the result, as mentioned in section III-A. This is confirmed by Fig. 3, where larger frame window width visibly leads to underestimating diffraction effects, as can be seen in sidelobe computation.

\section{B. Comparison with Fast Iterative Physical Optics results}

We present results obtained at wavelengths not smaller than $7 \mathrm{~cm}$, and for a simplified scenario involving only a maximum number of two reflections, as shown in Fig. 4, in order to make the problem amenable to Fast Iterative Physical Optics (FIPO). Fig. 5 compares FIPO and GBS results for directions scattered by faces 1 of both buildings. As observed in the previous comparison, GBS fits better to FIPO results for smaller values of $L$. The conclusion is that in case of multiple internal reflections GBS accuracy will be sufficient only at higher frequencies, for beams to be paraxial yet keeping sufficiently small footprints on "building walls", and limited enough transverse extent when propagating in "streets".

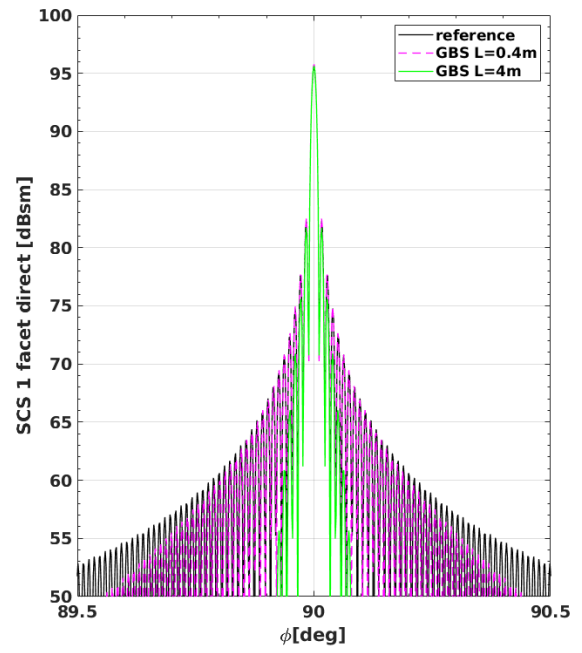

Fig. 3. Comparison of GBS result with analytical sinc solution, for two different $L$ parameter values.

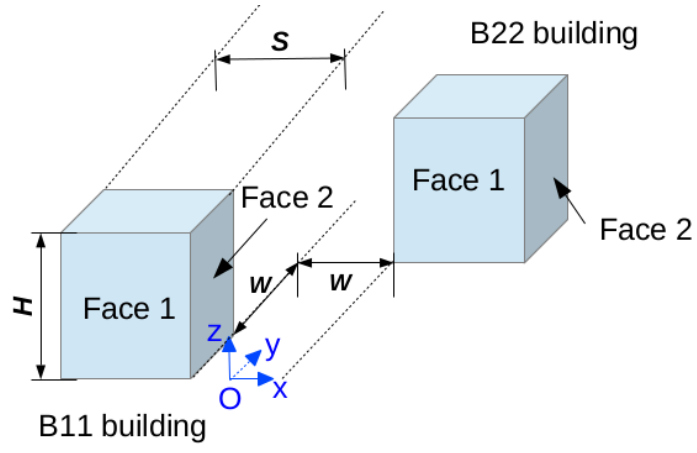

Fig. 4. Simplified scenario.

Fig. 6 compares FIPO and GBS results at $\lambda=10 \mathrm{~cm}$, for directions scattered after two reflections, either by Building 1 face 2 then Building 2 face 1, or vice versa.

\section{Computation time variation with problem size}

The monostatic SCS of the $2 \times 2$ "buildings" scenario of Fig. 1 is computed by our GBS algorithm first at $0.1 \mathrm{~m}$ wavelength, with the same frame parameters as in Fig. 2, then at $0.01 \mathrm{~m}$ wavelength (ten times smaller), with the same oversampling parameter, but with different choices for the $L$ parameter value. The various cases are listed in Table I, and corresponding CPU times indicated, as well as their variation with the parameter $N$, which is multiplied by 100 . The number of scattering directions was multiplied by 10 , i.e. $O\left(N^{1 / 2}\right)$, in order to keep the same sampling of the azimuth angles. The CPU time at the $0.1 \mathrm{~m}$ wavelength was less than $2 \mathrm{~s}$ for 10000 scattering directions, with $\approx 0.3 \mathrm{~s}$ only devoted to the first part of the algorithm (beam transfer), the rest of the time being devoted to field computation.

As can be seen in the table, variation of CPU time with problem size is highly dependent of the choice of $L$. Keeping 
TABLE I

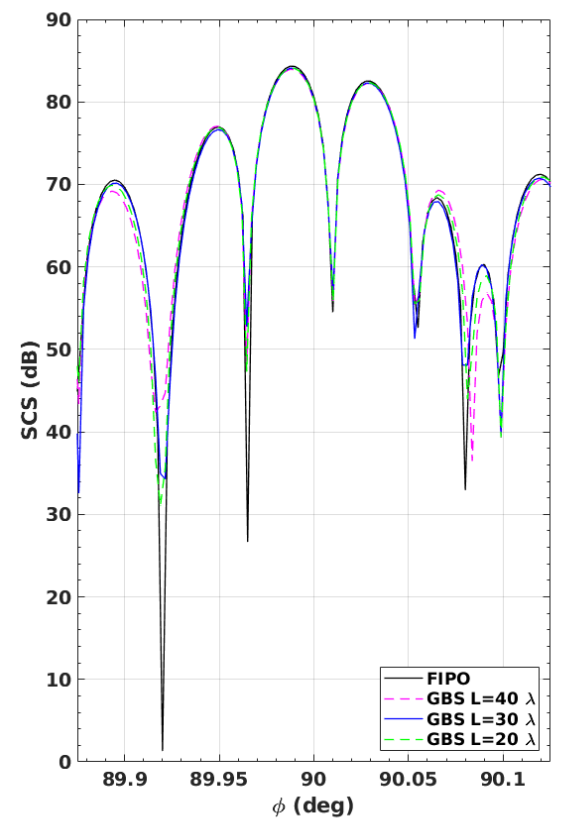

Fig. 5. Comparison between FIPO and GBS results at $\lambda=7 \mathrm{~cm}$, for scattering in directions close to the normal to building faces 1 .

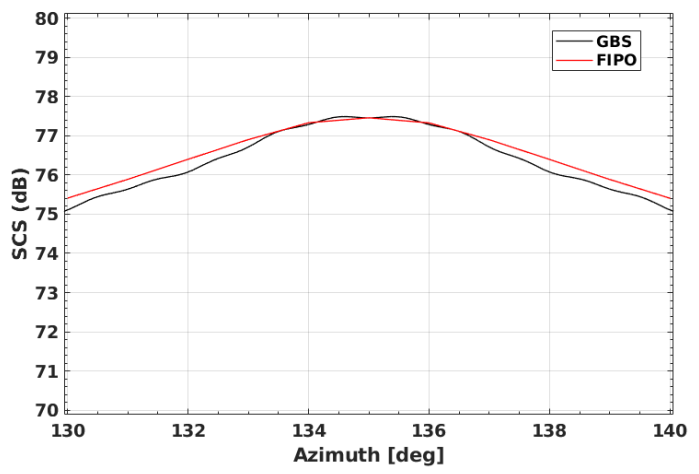

Fig. 6. Comparison between FIPO and GBS results at $\lambda=10 \mathrm{~cm}$, in directions scattered through two reflections.

the same $L$ value maintains the same CPU time, for a single scattering direction, for both wavelengths. Assuming that the GB width parameter is small enough as compared to walls and street dimensions, and that, at the lowest frequency of interest, the collimation distance is large enough to ensure beam collimation through all internal reflections, this choice is clearly the best one.

\section{CONCLUSION}

This work aims at testing the ability of a simple frame based GBS method to compute monostatic SCS of large environments involving multiple internal reflections. The method is clearly well suited for environments composed of planar surfaces, and first accuracy tests are encouraging. Testing the
GBS CPU TIMES AT $\lambda=0.01 \mathrm{M}$, FOR VARIOUS $L$ VALUES, VERSUS CPU TIME AT $\lambda=0.1 \mathrm{M}$

\begin{tabular}{|l|l|l|l|}
\hline $\boldsymbol{L}(\boldsymbol{m})$ & $\boldsymbol{v s} \mathbf{0 . 1} \boldsymbol{m}$ case $\boldsymbol{L}=\mathbf{4} \boldsymbol{m}$ & CPU time $(\boldsymbol{s})$ & CPU time vs $\boldsymbol{N}$ \\
\hline 4 & same $L$ & 11.3 & $O\left(N^{1 / 2}\right)$ \\
\hline 1.2 & same $b$ (isodiffracting) & 205 & $\approx O(N)$ \\
\hline 0.8 & & 611 & \\
\hline 0.4 & same $L / \lambda$ & 3884 & $>O\left(N^{3 / 2}\right)$ \\
\hline
\end{tabular}

method accuracy in cases when it would really be useful (problems with more internal reflections at higher frequencies) is a challenge as such problems can hardly be addressed by methods with reference accuracy.

\section{REFERENCES}

[1] D. Lugara, C. Letrou, A. Shlivinski, E. Heyman, and A. Boag, "Framebased Gaussian beam summation method: theory and application," Radio Science, vol. 38, no.2, pp. 8026-8040, April 2003.

[2] I. Gershenzon, Y. Brick, and A. Boag, "Shadow Radiation Iterative Physical Optics Method for High Frequency Scattering," IEEE Trans. Antennas and Propagation, vol. 66, pp. 871-883, Feb. 2018. 